\title{
Early intervention studies in infants and preschool children with cystic fibrosis: are we ready?
}

\author{
Stephen Stick ${ }^{1,12}$, Harm Tiddens $2,3,12$, Paul Aurora ${ }^{4}$, Per Gustafsson ${ }^{5}$, \\ Sarath Ranganathan ${ }^{6}$, Paul Robinson ${ }^{7}$, Margaret Rosenfeld ${ }^{8}$, Peter Sly ${ }^{9,10}$ \\ and Felix Ratjen ${ }^{11}$
}

\begin{abstract}
Affiliations: ${ }^{1}$ Dept of Respiratory Medicine, Princess Margaret Hospital for Children and University of Western Australia, Perth, ${ }^{6}$ Dept of Respiratory Medicine, Royal Children's Hospital Melbourne, Melbourne, ${ }^{7}$ Dept of Respiratory Medicine, The Children's Hospital at Westmead, Sydney, ${ }^{9}$ Queensland Children's Medical Research institute, The University of Queensland, Brisbane, and ${ }^{10}$ Dept of Respiratory Medicine, Royal Children's Hospital, Brisbane, Australia. ${ }^{2}$ Dept of Paediatric Respiratory Medicine, Erasmus MC - Sophia Children's Hospital, Rotterdam, and ${ }^{3}$ Dept of Radiology, Erasmus MC, Rotterdam, The Netherlands. "Paediatric Respiratory Medicine and Lung Transplantation, Great Ormond Street Hospital for Children NHS Foundation Trust, London, UK. ${ }^{5}$ Dept of Paediatrics, Central Hospital, Skövde, Sweden. ${ }^{8}$ Division of Pulmonary Medicine, Seattle Children's Hospital, Seattle, WA, USA. ${ }^{11}$ Division of Respiratory Medicine, Dept of Paediatrics, and Program in Physiology and Experimental Medicine, SickKids Research Institute, The Hospital for Sick Children, and University of Toronto, Toronto, ON, Canada. ${ }^{12}$ Both authors contributed equally to this manuscript.
\end{abstract}

Correspondence: S. Stick, Dept of Respiratory Medicine, Princess Margaret Hospital for Children, B0X D184 Perth, Western Australia 6005, Australia. E-mail: Stephen.Stickahealth.wa.gov

ABSTRACT Cystic fibrosis (CF) lung disease starts early in life and progresses even in the absence of clinical symptoms. Therefore, sensitive outcome measures to quantify and track these early abnormalities in infants and young children are needed; both for clinical care and interventional trials. Currently, the efficacy of most therapeutic interventions in CF has not been tested in children under the age of 6 years and drug development programmes have focused on assessing safety rather than efficacy in this age group. This article summarises the current status for outcome measures that can be utilised in clinical trials in infants and children with CF. Two methodologies are specifically highlighted in this review; chest computed tomography to assess structural damage of the lung and multiple breath washout as a technique to quantify ventilation inhomogeneity. While not all questions regarding the utility of these outcome measures in infants and young children have been resolved, significant advances have been made and it now appears feasible to design and conduct adequately powered efficacy studies in this age group. This could be a crucial step to further improve outcomes in CF patients as initiating effective treatment early is considered essential to prevent permanent lung damage.

@ERSpublications

Crucial to CF outcome is early effective treatment, hence intervention studies in infants and preschool children are a must http://ow.ly/ls $8 \mathrm{~Pa}$ 


\section{Introduction}

Cystic fibrosis (CF) lung disease begins early, with inflammation, infection and changes in structure and function often preceding respiratory symptoms $[1,2]$. As such the focus of therapeutic trials in early life needs to be on prevention of pulmonary abnormalities, rather than improving established disease. Given the recent success of CF transmembrane regulator (CFTR) directed therapy in CF, the ability to demonstrate its effect for disease prevention will be crucial to our understanding of the potential benefits of these therapies [3]. This poses a challenge of choosing appropriate outcome measures that can quantify and track early disease abnormalities in CF infants and young children. This article reviews the current knowledge of specific outcome measures, addresses their potential utilisation in clinical trials at present, and provides a framework of steps required to improve our knowledge about these variables in the future. The review is based on a research seminar organised by the European Respiratory Society and held on March 23-24, 2012 in Rotterdam, the Netherlands. The seminar sought to reach consensus regarding the practical implementation of these outcomes in clinical trials but not to determine the relative merits of the tests. However, during the course of the seminar discussions, two methods emerged that are particularly promising and close to clinical utilisation, namely computed tomography (CT) imaging of the lung and multiple-breath inert-gas washout testing to quantify ventilation inhomogeneity. Other areas discussed and included in this review were: bronchoalveolar lavage (BAL); infant and preschool lung function testing; pulmonary exacerbations; and systemic markers of inflammation. These latter areas are included in this review, not primarily as potential primary outcome variables for clinical trials but because they are likely to provide valuable information regarding mechanisms of drug action, or inform the clinical utility of the interventions being tested. The list of variables is not exclusive and other outcome measures may play an important role in individual studies. This review does not, therefore, claim to provide a complete summary of the field, but rather focuses on outcome measures that are closest to being considered for implementation in clinical trials.

Presently not all relevant scientific questions about the utility of any given outcome measure have been resolved. However, the authors feel strongly that unless we integrate outcome measures into clinical trials that provide insight into both pathological and physiological alterations and their modifications by therapy, paediatric investigational plans will not provide the information needed to understand the role of a given drug intervention in infants and young children with CF.

\section{Chest CT}

Lung imaging using CT is a sensitive method to detect and monitor structural changes relevant for patients of all ages with CF and is of particular relevance in infants and children $<6$ years of age, when structural disease is often very mild. The most important components of CF lung disease are bronchiectasis and airway obstruction causing air trapping. Chest CT is currently the most sensitive method and gold standard for the detection of bronchiectasis [4,5]. At birth CF patients have macroscopically normal lungs [6]. Bronchiectasis, which is an irreversible widening of bronchi, can develop in young children [7]. In cohort studies of children with CF diagnosed through newborn screening, bronchiectasis, trapped air and mucus impaction could be observed even in asymptomatic infants [8-11]. Bronchiectasis is a highly relevant endpoint in CF because it is: a risk factor for the development of respiratory tract exacerbations; associated with a lower health-related quality of life; and in end-stage lung disease the presence of bronchiectasis is associated with a higher mortality [12]. Importantly, the severity of CT detected structural changes on a CT scan correlates with markers of inflammation and infection in bronchoalveolar lavage fluid (BALF) $[8,11,13,14]$.

To quantify abnormalities, such as bronchiectasis and trapped air on a chest CT, robust scoring systems have been developed. The best validated scoring system is the CF-CT score, which is an upgraded system of the Brody II scoring system $[15,16]$. These manual scoring systems are relatively insensitive to technical differences caused by using different scan protocols and scanners and appear to be more sensitive than (infant) pulmonary function variables for detecting and monitoring the onset and progression of early CF lung disease $[8,14,17,18]$. Of all the structural changes that can be scored on a chest CT, bronchiectasis is the best validated and most reliable in young children [14]. Although air trapping is very common early on in children with CF [8], whether it represents or predicts the onset of irreversible structural lung disease is unknown. More sensitive quantitative semi-automated image analytic strategies for bronchiectasis and trapped air are in development. Currently these systems are time consuming and need to be automated.

Chest CT scores have been used as outcome variables in a limited number of intervention studies. CT scores improved with antibiotic treatment for a respiratory tract exacerbation in elderly subjects [19]. Similar findings were reported for paediatric patients $[13,20]$, and in small pilot studies CT scores improved after treatments, such as dornase alfa and tobramycin solution, were used for inhalation [21-25].

To date there have been no completed early intervention studies to prevent the onset of structural lung disease in infants newly diagnosed with CF. However, the ongoing COMBAT CF study (a randomised 
clinical trial of azithromycin to prevent bronchiectasis in infants, ClinicalTrials.gov identifier:NCT01270074) is the first to use chest CT as a primary endpoint and in this regard it is a prototype that could be considered a template for future studies from diagnosis aimed at preventing structural lung disease.

The advantages and disadvantages of using CT as an endpoint in young children are shown in table 1. The most important variable that requires standardisation during CT scanning is lung volume [26]. Most young children are not able to do a voluntary breath hold at the correct volume level and at the correct moment. Therefore, images have been obtained using sedation or anaesthesia to facilitate lung volume standardisation $[8,9,11]$. The anaesthetic protocol should include a recruitment procedure prior to image acquisition in order to avoid atelectasis, which is otherwise common in CT scans obtained during anaesthesia. One advantage of using an anaesthetic is the standardisation of the inspiratory lung volume that reduces both within-subject and between-subject variability for CT-derived outcome variables. Lately, fast CT scanners have been developed that allow for the acquisition of a chest CT during free tidal-volume breathing. The sensitivity of these chest-CT scans might be similar to that of a pressure-controlled expiratory chest CT; however, this needs to be further investigated. CT scans have the disadvantage of being associated with an increased exposure to ionizing radiation. Using a conservative computational model is was estimated that routine lifelong annual CT scans, starting at the age of 2 years, carries a low risk of lifelong radiation-induced mortality in CF patients [27]. In general routine chest CT scans are executed biannually, which reduces the estimated radiation risk by a half. Since the modelling study was executed, exposure doses have been further reduced with newer scanners and improved analysis algorithms. It is highly likely that this trend will continue. Clearly, when a chest CT of a child is taken, a state of the art lowdose protocol should be used, which enables the required information to be taken [28]. Clearly, scoring systems do not make full use of the rich digital information available in a CT image, which are labour intensive and subject to intra- and inter-subject variability. Hence, the development of automated systems that provide more sensitive, reliable and objective analysis of CT images suitable for multinational, multicentre clinical trials should be a priority.

\section{Multiple breath inert gas washout}

Multiple breath inert gas washout (MBW) tests assess the efficiency of ventilation distribution within the lungs (table 2). These tests describe the rate of clearance of a marker (or inert) gas from the lungs, or the uniformity of gas mixing within the time frame of a breath. A number of different inert gases can be used. Resident gases, such as nitrogen, can be washed out by breathing $100 \%$ oxygen, whilst non-resident gases, such as sulfur hexafluoride or helium $(\mathrm{He})$, require an additional wash-in phase after which they are washed out by breathing room air. As a tidal breathing test MBW is feasible across the paediatric age range [29-31]. Tests are typically performed in the supine position during quiet sleep in infants (with or without the use of sedation), and in a sitting upright and awake position from preschool age and onwards, using video distraction to encourage regular breathing during the test. The effect of sedation on test results is currently not well defined. Therefore, all studies within a single protocol should be undertaken under the same conditions, either with or without sedation and a mixture of study conditions must be avoided.

In addition to measured functional residual capacity (FRC), available indices of ventilation distribution efficiency from MBW can be divided into two categories: those describing global abnormalities (e.g. lung clearance index: LCI) and those providing more detailed insight into the relative contributions of the two main gas transport mechanisms within the lung, convection and diffusion. The latter are based on phase III slope analyses [32]. Phase III slope analysis indices are more complicated to calculate and require a greater

\section{TABLE 1 Advantages and disadvantages of chest computed tomography (CT) in young children}

\section{Advantages}

Most cystic fibrosis centres have a CT scanner

Feasible in all age groups

Gold standard to detect bronchiectasis

Bronchiectasis is well validated as clinical outcome measure

Lungs are normal at birth, at age 5 years half of the children have bronchiectasis

Trapped air reflecting small airways disease can be easily recognised on expiratory CT scan

Validated scoring systems for quantification are available

International standards for equipment requirements, procedures,

are in development

\section{Disadvantages}

lonising radiation limits repeatability to one chest CT every 1-2 years Need for general anaesthesia

Pressure-controlled volume procedure requires well trained teams

Costs

No validated automated system for image analysis available yet Centralised image analysis is currently required

Data transfer may be hampered by very large files 
level of quality control during testing than if assessing global measures, such as LCI, alone. This type of analysis is not currently feasible during infancy and has acceptable but not strong feasibility in younger children [33]. The advantage of phase III slope analysis is that it is thought to provide additional insight into the site of abnormality; although, such structure-function correlations require further confirmation in young children. Abnormalities in ventilation distribution may be generated more proximally, due to differences in ventilation among lung units with different time constants due to airway obstruction, or more distally, due to abnormalities in the region of the airways where convective and diffusive gas transport interact [32]. However, quality control is not yet standardised in any current MBW system and large between-test variability has been described even in healthy adults [34]. This report will focus on measures of global abnormalities, specifically LCI, for which more information is available in CF patients.

Research studies have shown that early on in the course of disease, marked abnormalities may be present in the uniformity of ventilation distribution in CF [29, 30, 35] and several other important progressive obstructive lung diseases [36, 37]. In CF lung disease, increased LCI values have been observed despite normal spirometry $[29,30,35]$. Increased LCI is associated with concurrent structural lung damage on CT scans in schoolchildren $[38,39]$ but the relations between LCI and irreversible structural changes are less clear in younger children [40]. There is recent evidence that MBW can track progressive early disease [41]. Importantly, MBW measured during the preschool age range is predictive for the development of abnormal spirometry later at early school age [42]. Intervention studies have also shown that significant improvements in LCI may be seen with treatments, such as nebulised hypertonic saline and dornase alfa, despite small patient numbers [43]. This combination of feasibility and robustness across wide age ranges, apparent sensitivity to detect early lung damage and subsequent change with interventions makes LCI an attractive potential tool for interventional studies.

Many of the studies mentioned above were performed using mass spectrometry and sulfur hexafluoride as a tracer gas, both of which are available in only a few centres. Reference data are available for sulfur hexafluoride MBW in infants $[44,45]$ and data assessing CF patients versus healthy controls across centres have been obtained in one study [46]. There is agreement within the CF community that nitrogen based systems are more likely to be suitable for multicentre studies. Currently, two recording systems are available commercially for use in children. At present the Ecomedics Exhalyzer D (Ecomedics AG, Duernten, Switzerland) is felt to be the most versatile commercial MBW device available. The Exhalyzer D has an infant module, which uses sulfur hexafluoride as the marker gas, but is not suitable for older age groups ( $>2$ years), and a nitrogen MBW module, which has been validated for use from late preschool to adult age (FRC 500-4000 mL) [47]. All the indices described previously are calculated by the Exhalyzer D system. Reference data in a large cohort are available for nitrogen MBW using this device (284 subjects aged 770 years; however, data are limited in infants and preschool children) [48]. The other commercial system available is the EasyOne Pro (ndd Medizintechnik AG, Zurich, Switzerland), which uses changes in molar mass $\left(\mathrm{g} \cdot \mathrm{mol}^{-1}\right)$ to monitor changes in inert gas concentration. MBW measurements with either sulfur hexafluoride or nitrogen have been validated, but only the latter of these two options is commercially available. The validation of nitrogen-based MBW FRC measurements over volumes ranging from 350 to $4000 \mathrm{~mL}$ [49] suggests its suitability for patients aged $\geqslant 2$ years and reference data are awaited. Available

TABLE 2 Advantages and disadvantages of the multiple breath washout (MBW) technique

Advantages

Performed during tidal breathing

Feasible in all age groups

$\mathrm{LCl}$ is more sensitive than spirometry indices to detect early CF lung disease

Global indices such as LCl correlate well with CT scores of lung pathology

Detects improvements in lung function with treatment interventions

Reference values are available for $\mathrm{LCl}$, and after infancy are very similar across childhood into early adulthood, facilitating longitudinal studies

International standards for equipment requirements, procedures, data analysis and quality control have been developed

\section{Disadvantages}

The utility of MBW as a surrogate for long-term outcome is not clearly defined

Most MBW studies have been undertaken using custom built recording systems, which complicates multicentre study feasibility.

Only a few commercial systems are marketed and there are few data in pre-school children using these systems.

Technology is currently not available in many CF centres

Lack of longitudinal data comparing $\mathrm{LCl}$ and chest CT

Sulfur hexafluoride is not approved in several countries, due to potential greenhouse effects and it may not be available as a medical gas

The use of $100 \%$ oxygen during nitrogen based MBW tests may affect breathing pattern in sedated infants

LCl: lung clearance index; CF: cystic fibrosis. 
indices include FRC and global measures of ventilation distribution, such as LCI, but phase III slope analysis is currently not available.

Utility in young children for both devices needs to be clarified. Further clinical studies are required on infants to determine the magnitude of the effect of $100 \%$ oxygen on breathing patterns and on MBW indices. More experience in preschool children is needed to document whether the high feasibility, previously demonstrated with sulfur hexafluoride based MBW, extends reference values into this younger age range. Equivalent reference data is also awaited.

If multicentre studies using MBW are to be undertaken, all centres must first gain experience with the method. Standardisation guidelines for inert gas washout are due to be published shortly that are applicable across all age ranges [50], Universal standard operating procedures (SOPs) must be adopted and applied across all centres. Initial studies in healthy children will allow assessment of adherence to these SOPs as LCI values obtained should fall within the range of published reference equations. In order to facilitate this, there is a need for "application specialists" that can train and supervise each centre.

\section{Infant lung function}

Infant lung function tests are currently performed in at least 115 centres worldwide. The raised volume rapid thoracoabdominal compression (RVRTC) technique is a "squeeze" technique adaptation that was introduced in the early 1990s and enabled the measurement of expired flows and volumes from full forced expiratory flow-volume curves, similar to those obtained using spirometry in older children (table 3) [51]. Guidelines were published by the American Thoracic Society (ATS)/European Respiratory Society (ERS) working group on infant and preschool lung function in 2005 [52], but many potential variations in performance of the technique and computational algorithms remain.

Three commercial systems are currently available: the nSpire Infant Pulmonary Laboratory (IPL; Longmont, CO, USA), the MasterScreen BabyBody (Jaeger, Hoechburg, Germany) and the Ecomedics device (Ecomedics AG). The Ecomedics device does not include a plethysmograph. Both the IPL and the MasterScreen BabyBody have been approved by the US Food and Drug Administration, but approval for the IPL device is limited to infants aged $>17$ weeks post-term, whereas the MasterScreen BabyBody is approved for use in preterm and term infants without any restriction to age or length. The Jaeger device is also Communauté Européenne marked. All systems are expensive and require intense training for their operators. Thus, for a study proposing an intervention as soon as possible after diagnosis by newborn screening (4-6 weeks) only the MasterScreen BabyBody has the necessary regulatory approval in the USA.

Infant pulmonary function testing is overall safe, but sleep (95\%), feeding (58\%) and behavioural changes $(57 \%)$ are commonly noticed by parents after the test, usually as a consequence of the sedation required to perform the procedure; however these changes are transient [53]. The reported feasibility of the RVRTC depends mainly on the success of achieving adequate sedation and varies from $72 \%$ up to $99 \%$ [53, 54]. Success in obtaining technically acceptable data depends on experience [54]. Mean within-test, withinsubject coefficients of variation range from $3.4 \%$ to $4.1 \%$ for forced expired volumes (FEV) and from $7.8 \%$ to $8.9 \%$ for forced expiratory flows (FEF) $[55,56]$. Measurements made 1 month apart in 41 infants with CF provide evidence for good reproducibility, with a mean intraclass correlation of 0.85 and $\geqslant 0.9$ for FEF and FEV, respectively [54]. No data on longer-term reproducibility are available.

Limited reference data exist for the RVRTC technique, published reference data are based on 155 healthy infants aged between 3 and 149 weeks using noncommercial homemade equipment [57]. When using the commercially available MasterScreen BabyBody, FEF/FEV data are significantly lower than the published reference data [58]. Optimally, in a clinical trial, the same device would be employed at all participating

\section{TABLE 3 Advantages and disadvantages of the raised volume rapid thoracoabdominal technique (RVRTC) technique}

\section{Advantages}

Guidelines for producing standardised RVRTC have been published Physiological concepts are related to those underpinning spirometry The test is as sensitive as multiple breath washout in infants with cystic fibrosis

Tracks through to school age

Commercial equipment available

Several centres perform RVRTC routinely

\section{Disadvantages}

\section{The requirement for sedation}

Expensive equipment

Time-consuming and technically-demanding test

Requirement for extensive training

Although the technique is standardised some issues such as computational algorithms, which differ in the commercial systems, have not been addressed so that measurements collected on different systems are not interchangeable Only experienced centres should be involved in clinical trials 
centres. An alternative would be to allow different devices, but have each centre test healthy infants in addition in order to evaluate inter-centre differences. The least favourable option would be to allow different devices with no healthy control data.

The utility of lung function studies to detect early abnormalities in infants with CF have been previously reviewed [59]. The RVRTC technique can identify diminished lung function in infants diagnosed both clinically [60] and by newborn screening [61]. Approximately $20 \%$ of infants with CF diagnosed clinically had diminished forced expiratory flow in $0.5 \mathrm{~s}$ (FEV0.5) compared with healthy infants without CF, but at least one parameter of FEF was diminished in up to 50\% [31]. Potential appropriate clinical trial endpoints may include FEV0.5 or mid-expiratory flow at $25-75 \%$ of forced vital capacity (MEF $25-75 \%$ ) but it was felt that, given the limited experience with infant pulmonary function tests as a clinical trial endpoint, choosing a single endpoint at the current time is premature.

In observational studies, lower lung function detected by the RVRTC technique was associated with pulmonary inflammation and declines, over time, in those with lower airway infection [1]. When RVRTC measurements were diminished in early infancy they appeared to track into later infancy [62], through the preschool years [63], and into school age where they correlated with lung function measured by spirometry [64]. However, because of the significant methodological differences in how the RVRTC is performed (sedation, supine position, positive pressure to raise lung volume to near total lung capacity) versus spirometry (awake, sitting or standing, negative pressure to reach total lung capacity), infant and schoolaged forced expiratory measurements are not directly comparable. Nevertheless, measurements have been shown to correlate with airway structural changes [10] and are inversely associated with the degree of neutrophilic inflammation and pathogen density in BAL [65].

There have been a few intervention studies in CF that have used infant lung function as an outcome [59]. The RVRTC technique has been used in two safety studies of hypertonic saline to demonstrate acute tolerability $[19,20,66,67]$. In a small study, of 11 infants and young children with CF, the RVRTC technique was used before and after treatment with intravenous antibiotics during pulmonary exacerbations and demonstrated a significant improvement in lung function following treatment,. These data suggest that lung function measurements are sensitive to changes in clinical status in this age group [68]. Estimates of the numbers of subjects required in early CF intervention studies in order to have $80 \%$ power to identify a difference in lung function at the $5 \%$ level of significance $(\alpha=0.05)$, based on a US observational multicentre study [54], suggest that 100 infants per study arm are required in order to demonstrate a difference of $14.4 \%$ in MEF25 (assuming an 18-month-old CF child with an average length). Because feasibility is $\sim 80 \%$ on each test occasion, $\sim 150$ infants per arm would need to be recruited. Thus, an eleven-centre study would need to recruit over three years if each centre were to recruit 10 subjects per year. However, RVTLC data from the recently completed Infant Study Inhaled Saline (ISIS) study would suggest that therapeutic effects can be observed with a lower number of infants in an interventional trial depending on the experience of participating centres [69].

In conclusion, the RVRTC technique is already being used in multicentre intervention studies and is an appropriate test to consider in future early CF trials. Training, experience and robust quality control are essential to enhance feasibility and ensure appropriate performance of the test. Data obtained using different equipment are not interchangeable and reference data for the commercial systems are urgently required but forthcoming. Workshop members felt strongly that a core centre should be established to develop and implement SOPs, train and certify sites, provide ongoing quality control, and perform central over-reading of all RVRTC tests so that only acceptable measurements are included in analytic data sets.

\section{Preschool lung function}

Spirometry is a term commonly used to describe the recording and interpretation of a maximal forced expiratory manoeuvre. It is the most commonly performed lung function test in adults and school age children, being used in lung function laboratories and also in the clinic and in the community. The most commonly reported measure is the forced expired volume in $1 \mathrm{~s}$ (FEV1). FEV1 is used extensively in the monitoring of individuals with CF and also as an outcome measure in epidemiological and intervention studies. In the preschool age group (aged 2-5 years), spirometry can be performed successfully in the great majority of children, though some modifications to the technique are required. In particular, the environment has to be child friendly and the operator has to be experienced in dealing with children. The approach is to create an environment closer to a play session, and to encourage the child to see the spirometry manoeuvre as a game. Online visual monitoring of the manoeuvre is essential and some form of incentive may be helpful. One commonly used strategy is to use training incentives prior to the spirometry manoeuvre, such as bubbles or whistles. In addition, many commercial spirometry packages include software that has computerised incentive games within them. Use of these games is not essential, but can be helpful in getting adequate results. 
There are clearly defined standards for quality control for spirometry for adults, which are also referenced for school age children [70]. It should be noted that these quality control standards are based upon limited data, particularly in school age children. The most important aspects of this quality control apply to the start of the test criteria, end of test criteria, and repeatability between measurements. In 2007 a joint ATS/ ERS working party published guidelines on performing spirometry in preschool subjects, which included modified quality control criteria [71]. For the start of the test, the back extrapolated volume should be $<12.5 \%$ of the forced vital capacity (FVC) or $80 \mathrm{~mL}$, whichever is the greater. For the end of the test, the only specified criterion is that cessation of flow must not occur at $>10 \%$ of the peak flow. A plateau on the volume-time curve in preschool subjects is rarely obtainable. For repeatability, ensuring that the second best manoeuvre is within $10 \%$ of the best is ideal. However, if only a single satisfactory manoeuvre can be obtained then these results should not be excluded simply because of poor repeatability. Rather there should be specific attention given to the acceptability of the best manoeuvre to see what the results from here can be reported. With regards to choice of outcome measure, many children at this age complete a forced expiration in $<1 \mathrm{~s}$, so FEV1 is not a valid outcome [72]. Forced expiratory volume in $0.75 \mathrm{~s}$ (FEV0.75) or $\mathrm{FEV} 0.5$ is recommended as an alternative. As for adult spirometry, the largest forced expiratory volume in $t \mathrm{~s}$ FEV $t$ and largest FVC from any usable curve should be reported as the best. These two outcomes do not need to come from the same curve. Expiratory flows should be taken from the expiration that has the largest sum of FEV $t$ and FVC, but it should be noted that all flows, but particularly late expiratory flows are highly dependent on the validity of FVC and the presence or absence of early termination [70, 71]. One study, from Lille (France), in preschool wheezers reported that the start of the test criteria above were obtained by a high proportion of preschool children in their laboratory and slightly tighter criteria may be appropriate [73]. However, these findings have not been confirmed by another centre to date.

Until recently a wide range of reference values were available for spirometry in children, some of which included the preschool age group. However, these reference equations were often based upon very limited data in preschool children and were not entirely consistent with each other. A comprehensive international collaboration has allowed the production of reference ranges for spirometry across all ages, published in 2008 [74]. The same group has also produced linked equations in forced spirometry in preschool children based upon almost 4000 observations collected from 15 centres over a 15-year period [75], which are recommended for use in this age group for all studies. It is known that the distribution of normal values across different ages is heteroscedastic (nonuniform), and for research purposes z-scores should be used in preference to per cent predicted values.

There are limited data at present comparing spirometry values in preschool children with CF with normal values. Cross-sectional studies suggest that, on measurement, preschool children with CF as a group have lower FEV $t$ than control children. However, only a small proportion (between $10 \%$ and $35 \%$ ), have FEV $t$ below the lower limit of normality $[29,76,77]$. There are very few data on the sensitivity of flows derived from preschool spirometry to detect early pulmonary abnormalities [63, 77]. Longitudinal data from infancy to preschool spirometry or preschool to school-age spirometry are also very limited. However, there is evidence of tracking of spirometry outcomes over this period [63], and also evidence that children who are infected with Pseudomonas aeruginosa early in life have persistently lower spirometry outcomes than their uninfected peers [63]. Data from the recent US multicentre data suggest that spirometry outcomes are more discriminative than outcomes from forced oscillation or inductance plethysmography [78].

As FEV1 is considered a primary measure of CF lung disease in older individuals, preschool spirometry could potentially serve as a meaningful outcome of early intervention studies involving preschool children. Presently, the following recommendations are made. 1) Preschool spirometry may be conducted using a variety of commercial devices. Minimum criteria for device acceptability include the ability to evaluate the flow-volume and volume-time curves in real time, accept forced expiratory manoeuvres $<1 \mathrm{~s}$ in duration, and report FEV0.5. Age-appropriate incentive software is helpful but not required. 2) Guidelines for preschool spirometry have been published [71] and any future trial SOP should be based upon these guidelines. 3) FEV0.5, FEV0.75 or FEF75 could all be appropriate clinical trial endpoints, but this will need to be verified further in interventional studies. 4) Sites joining a clinical trial network should be required to collect data on healthy preschool children prior to enrolling participants in CF clinical trials in order to assess intercentre variability. 5) As with other outcome measures, a core centre should be established to develop and implement SOPs, train and certify sites, provide ongoing quality control, and perform central over-reading of all tests so that only acceptable measurements are included in analytic data sets.

\section{Bronchoalveolar lavage}

$\mathrm{CF}$ lung disease is characterised by recurrent and persistent respiratory infections and inflammation dominated by neutrophils which is considered important in airway and lung damage. BAL offers the opportunity to sample lower airways directly. CF lung disease is heterogeneous in its distribution and BAL 
performed in one lobe may not be representative of disease activity in all areas of the lung. Despite these potential shortcomings longitudinal studies both in school age children and infants with CF have demonstrated that lower airway inflammation in CF is progressive and the Australian Respiratory Early Surveillance Team for Cystic Fibrosis (AREST CF) has shown that infection and inflammation are predictive of subsequent airway disease quantified by CT [14]. The major risk factors, in multivariate analyses, for the presence and progression of bronchiectasis in the AREST CF data are: active neutrophilic inflammation and the presence of free neutrophil elastase activity in BAL; and infection by P. aeruginosa $[11,14,79]$. While routine BAL to diagnose infection has not been shown to improve clinical outcome when targeted at improving detection, treatment and eradication of infection with P. aeruginosa [16], its accuracy of defining patients' infectious state, especially in relation to early infection with Staphylococcus aureus, is superior to other available culture techniques in non-sputum producing infants and young children [80]. While BAL in young children usually requires general anaesthesia, the overall risk of the procedure is low in CF patients [70].

BAL is unlikely to become a primary outcome measure in clinical trials in infants and young children. However, BAL can provide important mechanistic information in interventions that affect the balance if infection and inflammation. Examples from the COMBAT CF study (AZI001; ACTRN12610001072000; NCT01270074) are assessing the effect of azithromycin on lung disease progression in CF infants where BAL and these will provide important information on treatment efficacy on airway inflammation, bacterial and potentially viral infection as well as being a safety measure assessing the evolution of macrolide resistance of bacterial organisms. BAL can, therefore, support or define potential mechanisms of action that otherwise could not be clarified. In addition, BAL plays an important role in cross-validating other less invasive biomarkers that have the potential to be used as outcome measures derived from blood and urine, which could be sampled more frequently in an interventional trial. In trials of CFTR pharmacotherapy, while not essential for defining safety, BAL is currently the only way to demonstrate effects of therapy on the progression of airway inflammation. BAL could also give valuable information on changes in the airway microbiome, potentially positive or negative, during interventional studies.

While defining the mechanism of action is usually part of early phase studies, it is unlikely that full development programmes will happen in infants and young children and there could only be one pivotal study for any given drug in this age group. It may be important to integrate BAL into these trials; possibly in subgroups of patients. There could potentially be a disadvantage of performing BALs only at times of clinical stability as infection and inflammation and potential effects of interventions on these processes could be underestimated with this strategy. However, it is unlikely that a sampling strategy on BALs performed more frequently than once a year is realistic. Cross-validated systemic markers of inflammation and other culture techniques, to capture infection, may help to close the gap as they allow for more frequent sampling. BAL at times of clinical instability may increase its yield, but is also associated with a higher risk of procedure related side-effects.

Standardisation of BAL procedure has been proposed in the ERS Task Force report [81], but there is a need to update this document and this process is ongoing. By now, most centres perform BAL under general anaesthesia. Suction in the upper airway should be avoided to limit contamination with colonising bacteria. The need for additional local anaesthetics above the glottis is controversial, but using local anaesthetics below the vocal cords is not recommended as it can affect microbiological yield and is unlikely to be needed. While the ideal BAL protocol has not been defined, using $3 \times 1 \mathrm{~mL}$ per $\mathrm{kg}$ of body weight in a $20 \mathrm{~mL}$ syringes, in the middle lobe, is recommended as a standard procedure in CF (AREST CF SOP). In addition, a second sample that can be limited to $1 \mathrm{~mL}$ per $\mathrm{kg}$ of body weight should be sampled in the lingula or the most affected lobe, the latter to be defined by prior imaging or during the bronchoscopy procedure. Low pressure suction either manually or using wall suction is recommended. Whether to pool all samples for subsequent analysis or to use the first sample for microbiology only is still being debated and will be resolved in a study planned at the Rotterdam meeting. Details about the processing of the BAL and storage of the material for future studies are beyond the scope of this manuscript and will be addressed in other documents, such as the standard operating procedures developed by the Clinical Study Network of the European Cystic Fibrosis Society. The advantages and disadvantages of bronchoalveolar lavage are given in table 4.

\section{Pulmonary exacerbations}

Pulmonary exacerbations are a clinical endpoint (affecting how a person feels, functions or survives). Exacerbation rate is well established as an outcome measure in clinical trials of CF patients aged $>6$ years. These definitions usually capture events rate; there is currently no validated definition to capture the severity of pulmonary exacerbations. In participants aged $<6$ years, a definition using a combination of clinical signs and symptoms for a duration of at least 3-5 days that led to antibiotic therapy has been 
TABLE 4 Advantages and disadvantages of bronchoalveolar lavage (BAL)

Samples the lower airways directly

Superior to other techniques to obtain bacterial cultures Allows for assessment of airway inflammation

BAL defined inflammation predicts future bronchiectasis
Invasive, it requires general anaesthesia in this age group

Airway infection and inflammation is heterogeneous through the airways in cystic fibrosis patients

Short and long term variability for BAL markers has not been defined

Sampling during times of stability will underestimate the role of viral infections.

However, sampling during acute respiratory exacerbations will increase the risks for the procedure

developed and used in three clinical trials to date: the Early Pseudomonas Infection Control (EPIC) clinical trial [82], a trial of azithromycin in patients not chronically infected with $P$. aeruginosa [83] and the ISIS trial of hypertonic saline [69]. While a potentially promising endpoint, it has not been prospectively validated and, in contrast to exacerbations rates in older CF patients, its association with survival or longterm outcomes, such as lung function decline has not been evaluated. In addition, pulmonary exacerbations in infants and preschool age children are frequently triggered by viral respiratory infections, which are also common in the general population in this age range, and may not be reduced in frequency by therapies that enhance mucociliary clearance or are disease-modifying.

\section{Systemic markers of inflammation}

Ongoing neutrophilic inflammation in CF airways is present in most infants and young children with CF and best captured by BAL as outlined above. However, the invasiveness of the procedure limits repetitive use. Systemic markers of inflammation detected in either blood or urine could potentially be useful to monitor inflammation indirectly. So far, limited information is available in infants and young children, but studies in older children and adults have suggested that serum calprotectin and high sensitivity C-reactive protein (CRP) hold some promise as markers to track inflammation in CF. A recent study in CF patients with limited lung disease has shown that treatment with azithromycin reduced absolute neutrophil counts, serum calprotectin, serum amyloid A, and high sensitivity CRP when compared with patients receiving placebo [84]. Changes in calprotectin were also correlated to changes in lung function and weight both after 28 and 168 days of treatment. Additional studies in infants and young children are warranted to clarify the potential role of these and other surrogate markers of inflammation in interventional trials.

\section{Summary}

CF lung disease presents early in life, but symptoms are subtle and sensitive techniques are needed to capture and track these early abnormalities. A range of methods are now available to study the key features of CF lung disease ranging from technologies assessing structure and function to those focusing on infection and inflammation. We believe that it is unlikely that one method alone will become the "holy grail" technique to assess lung disease and quantify treatment response in infants and young children and, therefore, methodologies will need to vary depending on the intervention studied. At the present time, chest computer tomography and MBW based tests seems to be best suited for interventional trials in very young children with CF. While additional work is needed to clarify the role of these methodologies and to standardise their use, these efforts are well on their way. The authors of this report are of the opinion that studies of pharmacological interventions planned today should not be limited to assessing safety alone in infants and young children. The ultimate goal of therapy is not to provide short-term gains in function, but rather prevention of lung damage. This is best achieved by initiating therapy in early infancy, which is only going to be achieved by providing evidence of therapeutic efficacy through early intervention studies performed in this age group. Given the size of the target population and the requirement for multinational clinical trials we believe that it is imperative that a strategy to harmonise outcome variable methodologies across clinical trial networks, and to implement training and certification protocols, is undertaken. This will help the pharmaceutical industry plan studies for pipeline therapies and enhance research opportunities globally.

\section{References}

1 Pillarisetti N, Williamson E, Linnane B, et al. Infection, inflammation, and lung function decline in infants with cystic fibrosis. Am J Respir Crit Care Med 2011; 184: 75-81.

2 Sermet-Gaudelus I, Mayell SJ, Southern KW. Guidelines on the early management of infants diagnosed with cystic fibrosis following newborn screening. J Cyst Fibros 2010; 9: 323-329. 
Ramsey BW, Davies J, McElvaney NG, et al. A CFTR potentiator in patients with cystic fibrosis and the G551D mutation. N Engl J Med 2011; 365: 1663-1672.

4 Kang EY, Miller RR, Müller NL. Bronchiectasis: comparison of preoperative thin-section CT and pathologic findings in resected specimens. Radiology 1995; 195: 649-654.

Hansell DM. Bronchiectasis. Radiol Clin North Am 1998; 36: 107-128.

6 Gibson RL, Burns JL, Ramsey BW. Pathophysiology and management of pulmonary infections in cystic fibrosis. Am J Respir Crit Care Med 2003; 168: 918-951.

7 Bedrossian CW, Greenberg SD, Singer DB, et al. The lung in cystic fibrosis: a quantitative study including prevalence of pathologic findings among different age groups. Hum Pathol 1976; 7: 195-204.

8 Stick SM, Brennan S, Murray C, et al. Bronchiectasis in infants and preschool children diagnosed with cystic fibrosis after newborn screening. J Pediatr 2009; 155: 623-628.

9 Long FR. High-resolution computed tomography of the lung in children with cystic fibrosis: technical factors. Proc Am Thorac Soc 2007; 4: 306-309.

10 Martínez TM, Llapur CJ, Williams TH, et al. High-resolution computed tomography imaging of airway disease in infants with cystic fibrosis. Am J Respir Crit Care Med 2005; 172: 1133-1138.

11 Sly PD, Brennan S, Gangell C, et al. Lung disease at diagnosis in infants with cystic fibrosis detected by newborn screening. Am J Respir Crit Care Med 2009; 180: 146-152.

12 Loeve M, Hop WC, de Bruijne M, et al. Chest computed tomography scores are predictive of survival in patients with cystic fibrosis awaiting lung transplantation. Am J Respir Crit Care Med 2012; 185: 1096-1103.

13 Davis SD, Fordham LA, Brody AS, et al. Computed tomography reflects lower airway inflammation and tracks changes in early cystic fibrosis. Am J Respir Crit Care Med 2007; 175: 943-950.

14 Mott LS, Park J, Murray CP, et al. Progression of early structural lung disease in young children with cystic fibrosis assessed using CT. Thorax 2012; 67: 509-516.

15 Brody AS, Klein JS, Molina PL, et al. High-resolution computed tomography in young patients with cystic fibrosis: distribution of abnormalities and correlation with pulmonary function tests. J Pediatr 2004; 145: 32-38.

16 Wainwright CE, Vidmar S, Armstrong DS, et al. Effect of bronchoalveolar lavage-directed therapy on Pseudomonas aeruginosa infection and structural lung injury in children with cystic fibrosis: a randomized trial. JAMA 2011; 306 : $163-171$.

17 de Jong PA, Nakano Y, Lequin MH, et al. Progressive damage on high resolution computed tomography despite stable lung function in cystic fibrosis. Eur Respir J 2004; 23: 93-97.

18 de Jong PA, Lindblad A, Rubin L, et al. Progression of lung disease on computed tomography and pulmonary function tests in children and adults with cystic fibrosis. Thorax 2006; 61: 80-85.

19 Shah RM, Sexauer W, Ostrum BJ, et al. High-resolution CT in the acute exacerbation of cystic fibrosis: evaluation of acute findings, reversibility of those findings, and clinical correlation. AJR Am J Roentgenol 1997; 169: 375-380.

20 Brody AS, Molina PL, Klein JS, et al. High-resolution computed tomography of the chest in children with cystic fibrosis: support for use as an outcome surrogate. Pediatr Radiol 1999; 29: 731-735.

21 Robinson TE, Goris ML, Zhu HJ, et al. Dornase alfa reduces air trapping in children with mild cystic fibrosis lung disease: a quantitative analysis. Chest 2005; 128: 2327-2335.

22 Robinson TE, Leung AN, Northway WH, et al. Composite spirometric-computed tomography outcome measure in early cystic fibrosis lung disease. Am J Respir Crit Care Med 2003; 168: 588-593.

23 Nasr SZ, Kuhns LR, Brown RW, et al. Use of computerized tomography and chest x-rays in evaluating efficacy of aerosolized recombinant human DNase in cystic fibrosis patients younger than age 5 years: a preliminary study. Pediatr Pulmonol 2001; 31: 377-382.

24 Nasr SZ, Gordon D, Sakmar E, et al. High resolution computerized tomography of the chest and pulmonary function testing in evaluating the effect of tobramycin solution for inhalation in cystic fibrosis patients. Pediatr Pulmonol 2006; 41: 1129-1137.

25 Nasr SZ, Sakmar E, Christodoulou E, et al. The use of high resolution computerized tomography (HRCT) of the chest in evaluating the effect of tobramycin solution for inhalation in cystic fibrosis lung disease. Pediatr Pulmonol 2010; 45: 440-449.

26 Long FR, Williams RS, Adler BH, et al. Comparison of quiet breathing and controlled ventilation in the highresolution CT assessment of airway disease in infants with cystic fibrosis. Pediatr Radiol 2005; 35: 1075-1080.

27 de Jong PA, Mayo JR, Golmohammadi K, et al. Estimation of cancer mortality associated with repetitive computed tomography scanning. Am J Respir Crit Care Med 2006; 173: 199-203.

28 de Jong PA, Tiddens HA, Lequin MH, et al. Estimation of the radiation dose from CT in cystic fibrosis. Chest 2008; 133: 1289-1291.

29 Aurora P, Bush A, Gustafsson P, et al. Multiple-breath washout as a marker of lung disease in preschool children with cystic fibrosis. Am J Respir Crit Care Med 2005; 171: 249-256.

30 Gustafsson PM, Aurora P, Lindblad A. Evaluation of ventilation maldistribution as an early indicator of lung disease in children with cystic fibrosis. Eur Respir J 2003; 22: 972-979.

31 Lum S, Gustafsson P, Ljungberg H, et al. Early detection of cystic fibrosis lung disease: multiple-breath washout versus raised volume tests. Thorax 2007; 62: 341-347.

32 Robinson PD, Goldman MD, Gustafsson PM. Inert gas washout: theoretical background and clinical utility in respiratory disease. Respiration 2009; 78: 339-355.

33 Haidopoulou K, Lum S, Turcu S, et al. Alveolar LCI versus standard LCI in detecting early CF lung disease. Respir Physiol Neurobiol 2012; 180: 247-251.

34 Downie SR, Salome CM, Verbanck S, et al. Ventilation heterogeneity is a major determinant of airway hyperresponsiveness in asthma, independent of airway inflammation. Thorax 2007; 62: 684-689.

35 Fuchs SI, Sturz J, Junge S, et al. A novel sidestream ultrasonic flow sensor for multiple breath washout in children. Pediatr Pulmonol 2008; 43: 731-738.

36 Green K, Buchvald FF, Marthin JK, et al. Ventilation inhomogeneity in children with primary ciliary dyskinesia. Thorax 2012; 67: 49-53.

37 Gustafsson PM. Peripheral airway involvement in CF and asthma compared by inert gas washout. Pediatr Pulmonol 2007; 42: 168-176. 
38 Gustafsson PM, De Jong PA, Tiddens HA, et al. Multiple-breath inert gas washout and spirometry versus structural lung disease in cystic fibrosis. Thorax 2008; 63: 129-134.

39 Owens CM, Aurora P, Stanojevic S, et al. Lung Clearance Index and HRCT are complementary markers of lung abnormalities in young children with CF. Thorax 2011; 66: 481-488.

40 Hall GL, Logie KM, Parsons F, et al. Air trapping on chest CT is associated with worse ventilation distribution in infants with cystic fibrosis diagnosed following newborn screening. PLoS One 2011; 6: e23932.

41 Kieninger E, Singer F, Fuchs O, et al. Long-term course of lung clearance index between infancy and school-age in cystic fibrosis subjects. J Cyst Fibros 2011; 10: 487-490.

42 Aurora P, Stanojevic S, Wade A, et al. Lung clearance index at 4 years predicts subsequent lung function in children with cystic fibrosis. Am J Respir Crit Care Med 2011; 183: 752-758.

43 Amin R, Subbarao P, Jabar A, et al. Hypertonic saline improves the LCI in paediatric CF patients with normal lung function. Thorax 2010; 65: 379-383.

44 Fuchs SI, Gappa M. Lung clearance index: clinical and research applications in children. Paediatr Respir Rev 2011; 12: $264-270$

45 Lum S, Stocks J, Stanojevic S, et al. Age and height dependence of lung clearance index and functional residual capacity. Eur Respir J 2012; 41: 1371-1377.

46 Fuchs SI, Ellemunter H, Eder J, et al. Feasibility and variability of measuring the Lung Clearance Index in a multicenter setting. Pediatr Pulmonol 2012; 47: 649-657.

47 Singer F, Houltz B, Latzin P, et al. A realistic validation study of a new nitrogen multiple-breath washout system. PLoS One 2012; e36083.

48 Houltz B, Green K, Lindblad A, et al. Tidal $\mathrm{N}_{2}$ washout ventilation inhomogeneity indices in a reference population aged 7-70 years. Eur Respir J 2012; 40: Suppl. 56, 694s.

49 Anastassopoulou CG, Fuchs BB, Mylonakis E. Caenorhabditis elegans-based model systems for antifungal drug discovery. Curr Pharm Des 2011; 17: 1225-1233.

50 Robinson P, Latzin P, Verbanck S, et al. Guidelines for inert gas washout measurement using multiple and single breath tests. Eur Respir J 2013; 41: 507-513.

51 Turner DJ, Stick SM, Lesouef KL, et al. A new technique to generate and assess forced expiration from raised lung volume in infants. Am J Respir Crit Care Med 1995; 151: 1441-1450.

52 ATS/ERS statement: raised volume forced expirations in infants: guidelines for current practice. Am J Respir Crit Care Med 2005 172: 1463-1471.

53 Loland L, Bisgaard H. Feasibility of repetitive lung function measurements by raised volume rapid thoracoabdominal compression during methacholine challenge in young infants. Chest 2008; 133 : $115-122$.

54 Davis SD, Rosenfeld M, Kerby GS, et al. Multicenter evaluation of infant lung function tests as cystic fibrosis clinical trial endpoints. Am J Respir Crit Care Med 2010; 182: 1387-1397.

55 Mallol J, Aguirre VL, Wandalsen G. Variability of the raised volume rapid thoracic compression technique in infants with recurrent wheezing. Allergol Immunopathol (Madr) 2005; 33: 74-79.

56 Ranganathan SC, Hoo AF, Lum SY, et al. Exploring the relationship between forced maximal flow at functional residual capacity and parameters of forced expiration from raised lung volume in healthy infants. Pediatr Pulmonol 2002; 33: 419-428.

57 Jones M, Castile R, Davis S, et al. Forced expiratory flows and volumes in infants. Normative data and lung growth. Am J Respir Crit Care Med 2000; 161: 353-359.

58 Lum S, Hoo AF, Hulskamp G, et al. Potential misinterpretation of infant lung function unless prospective healthy controls are studied. Pediatr Pulmonol 2010; 45: 906-913.

59 Gappa M, Ranganathan SC, Stocks J. Lung function testing in infants with cystic fibrosis: lessons from the past and future directions. Pediatr Pulmonol 2001; 32: 228-245.

60 Ranganathan SC, Bush A, Dezateux C, et al. Relative ability of full and partial forced expiratory maneuvers to identify diminished airway function in infants with cystic fibrosis. Am J Respir Crit Care Med 2002; 166: 1350-1357.

61 Linnane BM, Hall GL, Nolan G, et al. Lung function in infants with cystic fibrosis diagnosed by newborn screening. Am J Respir Crit Care Med 2008; 178: 1238-1244.

62 Ranganathan SC, Stocks J, Dezateux C, et al. The evolution of airway function in early childhood following clinical diagnosis of cystic fibrosis. Am J Respir Crit Care Med 2004; 169: 928-933.

63 Kozlowska WJ, Bush A, Wade A, et al. Lung function from infancy to the preschool years after clinical diagnosis of cystic fibrosis. Am J Respir Crit Care Med 2008; 178: 42-49.

64 Harrison AN, Regelmann WE, Zirbes JM, et al. Longitudinal assessment of lung function from infancy to childhood in patients with cystic fibrosis. Pediatr Pulmonol 2009; 44: 330-339.

65 Peterson-Carmichael SL, Harris WT, Goel R, et al. Association of lower airway inflammation with physiologic findings in young children with cystic fibrosis. Pediatr Pulmonol 2009; 44: 503-511.

66 Dellon EP, Donaldson SH, Johnson R, et al. Safety and tolerability of inhaled hypertonic saline in young children with cystic fibrosis. Pediatr Pulmonol 2008; 43: 1100-1106.

67 Subbarao P, Balkovec S, Solomon M, et al. Pilot study of safety and tolerability of inhaled hypertonic saline in infants with cystic fibrosis. Pediatr Pulmonol 2007; 42: 471-146.

68 Pittman JE, Johnson RC, Davis SD. Improvement in pulmonary function following antibiotics in infants with cystic fibrosis. Pediatr Pulmonol 2012; 47: 441-446.

69 Rosenfeld M, Ratjen F, Brumback L, et al. Inhaled hypertonic saline in infants and children younger than 6 years with cystic fibrosis: the ISIS randomized controlled trial. JAMA 2012; 307: 2269-2277.

70 Miller MR, Hankinson J, Brusasco V, et al. Standardisation of spirometry. Eur Respir J 2005; 26: 319-338.

71 Beydon N, Davis SD, Lombardi E, et al. An official American Thoracic Society/European Respiratory Society statement: pulmonary function testing in preschool children. Am J Respir Crit Care Med 2007; 175: $1304-1345$.

72 Aurora P, Gustafsson P, Bush A, et al. Multiple breath inert gas washout as a measure of ventilation distribution in children with cystic fibrosis. Thorax 2004; 59: 1068-1073.

73 Neve V, Edme JL, Devos P, et al. Spirometry in 3-5-year-old children with asthma. Pediatr Pulmonol 2006; 41: 735-743.

74 Stanojevic S, Wade A, Stocks J, et al. Reference ranges for spirometry across all ages: a new approach. Am J Respir Crit Care Med 2008; 177: 253-260. 
Stanojevic S, Wade A, Cole TJ, et al. Spirometry centile charts for young Caucasian children: the Asthma UK Collaborative Initiative. Am J Respir Crit Care Med 2009; 180: 547-552.

76 Marostica PJ, Weist AD, Eigen H, et al. Spirometry in 3- to 6-year-old children with cystic fibrosis. Am J Respir Crit Care Med 2002; 166: 67-71.

77 Vilozni D, Bentur L, Efrati O, et al. Spirometry in early childhood in cystic fibrosis patients. Chest 2007; 131: 356-361.

78 Kerby GS, Rosenfeld M, Ren CL, et al. Lung function distinguishes preschool children with CF from healthy controls in a multi-center setting. Pediatr Pulmonol 2012; 47: 597-605.

79 Stick SM, Brennan S, Murray C, et al. Bronchiectasis in infants and preschool children diagnosed with cystic fibrosis after newborn screening. J Pediatr 2009; 155: 623-628e1.

80 Rosenfeld M, Emerson J, Accurso F, et al. Diagnostic accuracy of oropharyngeal cultures in infants and young children with cystic fibrosis. Pediatr Pulmonol 1999; 28: 321-328.

81 de Blic J, Midulla F, Barbato A, et al. Bronchoalveolar lavage in children. Eur Respir J 2000; 15: 217-231.

82 Treggiari MM, Retsch-Bogart G, Mayer-Hamblett N, et al. Comparative efficacy and safety of 4 randomized regimens to treat early Pseudomonas aeruginosa infection in children with cystic fibrosis. Arch Pediatr Adolesc Med 2011; 165: 847-856.

83 Saiman L, Anstead M, Mayer-Hamblett N, et al. Effect of azithromycin on pulmonary function in patients with cystic fibrosis uninfected with Pseudomonas aeruginosa: a randomized controlled trial. JAMA 2010; 303: 1707-1015.

84 Ratjen F, Saiman L, Mayer-Hamblett N, et al. Effect of azithromycin on systemic markers of inflammation in cystic fibrosis patients uninfected with Pseudomonas aeruginosa. Chest 2012; 142: 1259-1266. 